\title{
A k-OUT-OF-n RELIABILITY SYSTEM WITH AN UNRELIABLE SERVER AND PHASE TYPE REPAIRS AND SERVICES: THE (N,T) POLICY
}

\author{
SRINIVAS R. CHAKRAVARTHY \\ Kettering University \\ Department of Industrial and Manufacturing Engineering $\&$ Business \\ Flint, MI 48504, US \\ E-mail: schakrav@kettering.edu
}

\author{
A. KRISHNAMOORTHY ${ }^{1}$ and P.V. USHAKUMARI ${ }^{2}$ \\ Cochin University of Science and Technology \\ Department of Mathematics \\ Cochin 682022, India \\ E-mail: ak@cusat.ac.in
}

(Received June, 2001; Revised October, 2001)

In this paper we study a $k$-out-of- $n$ reliability system in which a single unreliable server maintains $n$ identical components. The reliability system is studied under the $(N, T)$ policy. An idle server takes a vacation for a random amount of time $T$ and then attends to any failed component waiting in line upon completion of the vacation. The vacationing server is recalled instantaneously upon the failure of the $N^{t h}$ component. The failure times of the components are assumed to follow an exponential distribution. The server is subject to failure with failure times exponentially distributed. Repair times of the component, fixing times of the server, and vacationing times of the server are assumed to be of phase type. Using matrix-analytic methods we perform steady state analysis of this model. Time spent by a failed component in service, total time in the repair facility, vacation time of the server, non-vacation time of the server, and time until failure of the system are all shown to be of phase type. Several performance measures are evaluated. Illustrative numerical examples are presented.

Key words: Reliability System, $k$-out-of- $n$ System, $(N, T)$-Policy, Phase Type Distribution, Matrix-Analytic Methods, Algorithmic Probability, and Optimization.

AMS subject classifications: $90 \mathrm{~B} 25,60 \mathrm{~K} 25$.

\footnotetext{
${ }^{1}$ Research in part supported by UGC, India.

${ }^{2}$ Research in part supported by CSIR, India. 


\section{Introduction}

When dealing with critical systems such as aircrafts, space shuttles, nuclear plants, satellites, electric generators, and computer systems, it is imperative that redundancy be in the system. The redundancy is in the form of multiple (often identical) components connected in such a way that when on component fails the others not only keep the system functioning but share the increased load due to fewer operating components. Also, redundancy is highly cost effective in achieving a certain reliability level of the system. A common form of redundancy is a $k$-out-of- $n$ system in which at least $k$ components of $n$ must be functioning for the system to be operational. $k$-outof- $n$ reliability systems have been studied extensively in the context of computing the reliability, optimization of the system, common cause failures, and repair facility availability for fixing failed components $[1-4,7-10,13-18,20,21]$. Though there are many papers on such systems, very few papers address load-sharing $k$-out-of- $n$ systems $[12,19]$. Load-sharing systems are natural and very useful in dealing with systems such as nuclear plants, aircraft engines, electric generators, computer systems, suspension bridge cables and other workload balancing systems.

When analyzing the reliability of systems with a repair facility to fix failed components (or machines), a review of past work on machine repairman problems shows two distinct areas of research:

(1) systems with reliable servers and

(2) systems with unreliable servers

Most of the research addressing machine repairman problems has focused on reliable servers Research work dealing with unreliable servers is limited in the literature [6, 11, 22-29]. Recently a comparative analysis of steady state performance measures under a variety of distributions for repair times and service times for a 1-out-of- $n$ reliability system with a single unreliable repairman was given in Chakravarthy and Agarwal [6].

In this paper, we will extend the work of [6] for a $k$-out-of- $n$ system in which

(a) the server is unreliable,

(b) the failed server is fixed by a repair facility,

(c) the server goes on vacation for a random amount of time whenever the system is functioning with all $n$ components,

(d) upon completing a vacation, the server goes for another vacation of random duration when there are no failed components waiting to be fixed, and

(e) the vacationing server is recalled instantaneously when the number of failed components reach a predetermined threshold $N$ assumed to be less than or equal to $n-k$.

We consider a $k$-out-of- $n$ system that can be cold, warm, or hot.

This paper is organized as follows. In Section 2, we describe the model under study and give a brief review of PH-distributions. The Markov chain description of the three reliability systems is presented in Section 3. The steady state analysis of the systems is presented in Section 4. The performance measures for studying the qualitative behavior of the reliability systems are developed in Section 5, and a few representative numerical examples are discussed in Section 6. 


\section{Model Description}

We consider a $k$-out-of- $n$ load-sharing reliability system with $n$ identical components which can fail independently of each other, and a single unreliable server that attends to failed components. The system will be functional if and only if there are $k$ or more working components. Failure times of the components are assumed to be exponential with a parameter that depends on the number of working components. That is, if there are $i$ working components, then the failure times are exponential with parameter $\lambda_{i}$.

Upon failure, a component enters into repair facility and the repair times follow a PH-distribution with representation $\left(\beta_{1}, S_{1}\right)$ of of order $m_{1}$. When the server is busy or when the server is unavailable, the failed component joins the queue. Once repaired, the component is returned back to the system as new.

It is assumed that the server is subject to failure and the failure times of the server are assumed to follow an exponential distribution with parameter $\mu_{S F}$. The fixing times of the server follow a PH-distribution with representation $\left(\beta_{2}, S_{2}\right)$ of order $m_{2}$. Once fixed, the server resumes the work of failed component at the stage where it left servicing the failed component.

Whenever there is no component waiting for repair, the server takes a vacation for a random time $T$ having a Ph-distribution with representation $\left(\beta_{3}, S_{3}\right)$ of order $m_{3}$.

We consider a $k$-out-of- $n$ reliability system that is cold, warm, or hot. By a cold system, we mean a reliability system in which the individual components cease to fail upon system failure. Note that the system failure occurs as soon as the number of functioning components drops below $k$. By a warm system, we mean a system in which the individual components continue to fail, but with a smaller failure rate, even after a system failure. In a hot system, individual components continue to fail at the same rate after a system failure.

In the sequel, we will assume that the failure rate of components is inversely proportional to the number of functioning components. That is, if there are $i$ functioning components, then $\lambda_{i}=\lambda / i$. Note that for a cold system, $\lambda_{i}=\lambda / i$, for $1 \leq i \leq n-k, \lambda_{i}=0$, for $n-k+1 \leq i \leq n$. For a warm system, $\lambda_{i}=\lambda / i$, for $1 \leq$ $i \leq n-k, \lambda_{i}=\theta / i$, for $n-k+1 \leq \bar{i} \leq n$, where $\theta<\lambda$. In the case of hot system, $\lambda_{i}=\lambda / i$, for $1 \leq i \leq n$. For the sake of simplicity, we will make this assumption, though the model is valid for the general case of the failure rate. In the following, $e$ and $I$ will represent a column vector of 1 's, and the identity matrix of appropriate dimensions, respectively. The notation $\otimes$ will stand for the Kronecker product of two matrices. Thus, if $A$ is an $m \times n$ matrix and if $B$ is a $p \times q$ matrix, then $A \otimes B$ will denote a $m p \times n q$ matrix whose $(i, j)^{t h}$ block matrix is given by $a_{i j} B$. For more details on Kronecker products, we refer the reader to [5].

Before we describe the Markov chain of the repairman model, we present a review of PH-distributions.

Phase type distributions: Poisson arrival processes and exponential service time distributions have mathematical properties that make queueing models very attractive and tractable. However, in applications these assumptions are highly restrictive. To remove some of the restrictions, Neuts [13] developed the theory of PH-distributions and related point processes. In stochastic modeling, $\mathrm{PH}$-distributions lend themselves naturally to algorithmic implementation and have nice closure properties with a related matrix formalism that make them attractive for practical use. In this section we review continuous-time PH-distributions. 
Continuous-time PH-distributions: Consider a finite Markov chain (MC) with $m$ transient states and one absorbing state with the infinitesimal generator $Q$ partitioned as

$$
Q=\left[\begin{array}{cc}
S & S^{\mathbf{0}} \\
\mathbf{0} & 0
\end{array}\right],
$$

where $S$ is a matrix of order $m$ and $S^{\mathbf{0}}$ is a column vector such that $S e+S^{\mathbf{0}}=0$. The vector $e$ is a column of 1's. It is necessary and sufficient that $S$ be nonsingular for eventual absorption into the absorbing state, starting from any initial state. Suppose that the initial state of the MC is chosen according to the probability vector $\left(\beta, \beta_{m+1}\right)$ and let $X$ denote the time until absorption. Then $X$ is a continuous random variable on nonnegative values with a probability distribution function $F(x)$ given by $F(x)=1-\beta e^{S x}$ e for $x \geq 0$.

Note that

(a) the $i^{\text {th }}$ component of the vector $e^{S x} e$ is the probability that starting in state $i$, absorption has not occurred by time $x$;

(b) the $(i, j)^{t h}$ component of the matrix $(-S)^{-1}$ is the conditional expected total time spent in state $j$ before absorption given that the initial state is $i$.

The function $F(\cdot)$ has a jump $\beta_{m+1}$ at the origin, but in most cases, $F(0)=0=$ $\beta_{m+1}$. Such a probability function constructed from a finite MC with a single absorbing state is a continuous $\mathrm{PH}$-distribution. The pair $(\beta, S)$ of order $m$ is said to be a representation of the $\mathrm{PH}$-distribution.

The transition matrix $P(x)=e^{Q x}$ is of the form

$$
e^{Q x}=\left[\begin{array}{cc}
e^{S x} & e-e^{S x} e \\
0 & 1
\end{array}\right]
$$

suggesting that the density $f(x)$ of $F(x)$ is either identically 0 or strictly positive for all $x>0$ (see [5]). In the latter case, $f(x)$ is given by $f(x)=\beta e^{S x} S^{0}$, for $x \geq 0$. The $k^{\text {th }}$ noncentral moment of $F(x)$ is given by

$$
\mu_{k}^{\prime}=k ! \alpha(-S)^{-k} e, \text { for } k \geq 0 \text {. }
$$

When $m=1, S=-\lambda$, the underlying PH-distribution becomes exponential. A generalized Erlang distribution of order $m$ is a PH-distribution with representation $(\beta, S)$

$$
\beta=(1,0, \ldots, 0) \text { and } S=\left[\begin{array}{ccccc}
-\lambda_{1} & \lambda_{1} & 0 & \ldots & 0 \\
0 & -\lambda_{2} & \lambda_{2} & \ldots & 0 \\
\vdots & \vdots & \vdots & \ldots & \vdots \\
0 & 0 & 0 & \ldots & \lambda_{m-1} \\
0 & 0 & 0 & \ldots & -\lambda_{m}
\end{array}\right] \text {. }
$$

A hyperexponential distribution of order $m$ is a $\mathrm{PH}$-distribution with representation $(\beta, S)$ 


$$
\beta=\left(\beta_{1}, \beta_{2}, \ldots, \beta_{m}\right) \text { and } S=\left[\begin{array}{ccccc}
-\lambda_{1} & 0 & 0 & \ldots & 0 \\
0 & -\lambda_{2} & 0 & \ldots & 0 \\
\vdots & \vdots & \vdots & \ldots & \vdots \\
0 & 0 & 0 & \ldots & 0 \\
0 & 0 & 0 & \ldots & -\lambda_{m}
\end{array}\right]
$$

For any PH-distribution, there exist infinitely many different representations. For example, if $\beta=u$ where $u$ is the left eigenvector of $S$ (with $u$ normalized to be a probability vector) corresponding to the eigenvalue $-\eta$ of maximum real part, then the representation $(u, S)$ of order $m$ is an exponential distribution with parameter $\eta$.

\section{The Markov Process}

The model outlined in Section 1 can be described by a Markov process. The state space of the Markov process depends on whether the system is cold, warm or hot. In the case of a cold reliability system, the state space is $\Omega_{c}=\left\{\left\{\left\{\left(i, j_{3}\right): 1 \leq j_{3} \leq m_{3}\right\}\right.\right.$, $\left.0 \leq i \leq N-1\} \cup\left\{\left(i, j_{1}, j_{2}\right): 1 \leq j_{1} \leq m_{1}, 1 \leq j_{2} \leq m_{2}\right\}, 1 \leq i \leq n-k+1\right\} \cup\left\{\left\{\left\{\left(i, j_{1}\right):\right.\right.\right.$ $\left.\left.\left.1 \leq j_{1} \leq m_{1}\right\}, 1 \leq i \leq n-k+1\right\}\right\}$. For the other two cases, the state space is $\Omega_{w h}=$ $\left\{\left\{\left\{\left(i, j_{3}\right): 1 \leq j_{3} \leq m_{3}\right\}, 0 \leq i \leq N-1\right\} \cup\left\{\left(i, j_{1}, j_{2}\right): 1 \leq j_{1} \leq m_{1}, \quad 1 \leq j_{2} \leq m_{2}\right\}, \quad 1 \leq i\right.$ $\leq n\} \cup\left\{\left\{\left\{\left(i, j_{1}\right): 1 \leq j_{1} \leq m_{1}\right\}, 1 \leq i \leq n\right\}\right\}$. The sets of states $i^{*}=\left\{\left(i, j_{3}\right): 1 \leq\right.$ $\left.j_{3} \leq m_{3}\right\}$ corresponds to the case where $i$ components are under repair and the server is on vacation in phase $j_{3}$. The set of states $i^{\prime}=\left\{\left(i, j_{1}, j_{2}\right): 1 \leq j_{1} \leq m_{1}, 1 \leq j_{2} \leq\right.$ $\left.m_{2}\right\}$ corresponds to the case where $i$ components and the server are under repair, the server repair in phase $j_{2}$, and the server failed while attending a failed component in phase $j_{1}$. The set of states $i=\left\{\left(i, j_{1}\right): 1 \leq j_{1} \leq m_{1}\right\}$ corresponds to the case where $i$ components are under repair and the current service is in phase $j_{1}$.

The Markov process for a cold $k$-out-of- $n$ reliability system has the infinitesimal generator $Q_{c}$ given by

$$
Q_{c}=\left[\begin{array}{ccc}
B_{11}^{c} & B_{12}^{c} & 0 \\
B_{21}^{c} & B_{22}^{c} & \mu_{S F}\left(I \otimes \beta_{2}\right) \\
0 & I \otimes S_{2}^{0} & B_{33}^{c}
\end{array}\right],
$$

where

$$
\begin{aligned}
& B_{11}^{c}= \\
& {\left[\begin{array}{cccccc}
S_{3}+S_{3}^{0} \beta_{3}-\lambda I & \lambda I & 0 & \ldots & 0 & 0 \\
0 & S_{3}-\lambda I & \lambda I & \ldots & 0 & 0 \\
\vdots & \vdots & \vdots & \ldots & \vdots & \vdots \\
0 & 0 & 0 & \ldots & S_{3}-\lambda I & \lambda I \\
0 & 0 & 0 & \ldots & 0 & S_{3}-\lambda I
\end{array}\right]}
\end{aligned}
$$




$$
\begin{aligned}
& B_{12}^{c}=\left[\begin{array}{ccccccccc}
0 & 0 & 0 & \ldots & 0 & 0 & \ldots & 0 & 0 \\
S_{3}^{0} \beta_{1} & 0 & 0 & \ldots & 0 & 0 & \ldots & 0 & 0 \\
0 & S_{3}^{0} \beta_{1} & 0 & \ldots & 0 & 0 & \ldots & 0 & 0 \\
\vdots & \vdots & \vdots & \ldots & \vdots & \vdots & \ldots & \vdots & \vdots \\
0 & 0 & 0 & \ldots & 0 & 0 & \ldots & 0 & 0 \\
0 & 0 & 0 & \ldots & S_{3}^{0} \beta_{1} & \lambda e \beta_{1} & \ldots & 0 & 0
\end{array}\right] \\
& B_{21}^{c}=\left[\begin{array}{ccccc}
S_{1}^{0} \beta_{3} & 0 & 0 & \ldots & 0 \\
0 & 0 & 0 & \ldots & 0 \\
\vdots & \vdots & \vdots & \ldots & \vdots \\
0 & 0 & 0 & \ldots & 0 \\
0 & 0 & 0 & \ldots & 0
\end{array}\right]
\end{aligned}
$$

with

$$
B_{22}^{c}=\left[\begin{array}{cccccc}
A_{1} & \lambda I & 0 & \ldots & 0 & 0 \\
S_{1}^{0} \beta_{1} & A_{1} & \lambda I & \ldots & 0 & 0 \\
0 & S_{1}^{0} \beta_{1} & A_{1} & \ldots & 0 & 0 \\
\vdots & \vdots & \vdots & \ldots & \vdots & \vdots \\
0 & 0 & 0 & \ldots & S_{1}^{0} \beta_{1} A_{1}+\lambda I
\end{array}\right]
$$

The Markov process for a warm $k$-out-of- $n$ reliability system has the infinitesimal generator $Q_{w}$ given by

$$
Q_{w}=\left[\begin{array}{ccc}
B_{11}^{c} & B_{12}^{w} & 0 \\
B_{21}^{w} & B_{22}^{w} & \mu_{S F}\left(I \otimes \beta_{2}\right) \\
0 & I \otimes S_{2}^{0} & B_{33}^{w}
\end{array}\right],
$$

where the $B_{i j}$ 's are such that $B_{12}^{w}$ and $B_{21}^{w}$ are of the form $B_{12}^{c}$ and $B_{21}^{c}$ except that the dimensions of these matrices are, respectively, $\mathrm{Nm}_{3} \times n m_{1}$ and $n m_{1} \times N m_{3}$. The matrices $B_{22}^{w}$ and $B_{33}^{w}$ are given by 
$B_{22}^{w}=\left[\begin{array}{ccccccccccc}A_{1} & \lambda I & 0 & \ldots & 0 & 0 & 0 & 0 & \ldots & 0 & 0 \\ S_{1}^{0} \beta_{1} & A_{1} & \lambda I & \ldots & 0 & 0 & 0 & 0 & \ldots & 0 & 0 \\ 0 & S_{1}^{0} \beta_{1} & A_{1} & \ldots & 0 & 0 & 0 & 0 & \ldots & 0 & 0 \\ \vdots & \vdots & \vdots & \ldots & \vdots & \vdots & \vdots & \vdots & \ldots & \vdots & \vdots \\ 0 & 0 & 0 & \ldots & S_{1}^{0} \beta_{1} & A_{3} & \theta I & 0 & \ldots & 0 & 0 \\ 0 & 0 & 0 & \ldots & 0 & S_{1}^{0} \beta_{1} & A_{3} & \theta I & \ldots & 0 & 0 \\ \vdots & \vdots & \vdots & \ldots & \ldots & \vdots & \vdots & \vdots & \ldots & \vdots & \vdots \\ 0 & 0 & 0 & \ldots & 0 & 0 & 0 & 0 & \ldots & S_{1}^{0} \beta_{1} A_{3}+\theta I\end{array}\right]$

$$
B_{33}^{w}=\left[\begin{array}{cccccccccc}
A_{2} & \lambda I & 0 & \ldots & 0 & 0 & 0 & \ldots & 0 & 0 \\
0 & A_{2} & \lambda I & \ldots & 0 & 0 & 0 & \ldots & 0 & 0 \\
0 & 0 & A_{2} & \ldots & 0 & 0 & 0 & \ldots & 0 & 0 \\
\vdots & \vdots & \vdots & \ldots & \vdots & \vdots & \vdots & \ldots & \vdots & \vdots \\
0 & 0 & 0 & \ldots & A_{4} & \theta I & 0 & \ldots & 0 & 0 \\
0 & 0 & 0 & \ldots & 0 & A_{4} & \theta I & \ldots & 0 & 0 \\
\vdots & \vdots & \vdots & \ldots & \vdots & \vdots & \vdots & \ldots & \vdots & \vdots \\
0 & 0 & 0 & \ldots & 0 & 0 & 0 & \ldots & 0 & A_{4}+\theta I
\end{array}\right]
$$

with

$$
A_{3}=S_{1}-\left(\theta+\mu_{S F}\right) I \text { and } A_{4}=I \otimes S_{2}-\theta I
$$

The Markov process for a hot $k$-out-of- $n$ reliability system has the infinitesimal generator $Q_{h}$ given by

$$
Q_{h}=\left[\begin{array}{ccc}
B_{11}^{c} & B_{12}^{h} & 0 \\
B_{21}^{h} & B_{22}^{h} & \mu_{S F}\left(I \otimes \beta_{2}\right) \\
0 & I \otimes S_{2}^{0} & B_{33}^{h}
\end{array}\right],
$$

where $B_{12}^{h}, B_{21}^{h}, B_{22}^{h}$ and $B_{33}^{h}$ are of the form $B_{12}^{w}, B_{21}^{w}, B_{22}^{w}$ and $B_{33}^{w}$, except that $\theta$ in these matrices is replaced by $\lambda$.

\section{The Steady-State Analysis}

In this section we will present the steady-state analysis of the reliability system discussed above. 


\subsection{The Steady-State Probability Vector for the Cold System}

The steady-state equations are obtained by solving $x Q_{c}=0$, and $x e=1$. By partitioning $x=\left(u_{0}, u_{1}, \ldots, u_{N-1}, v_{1}, v_{2}, \ldots, v_{n-k+1}, w_{1}, w_{2}, \ldots, w_{n-k+1}\right)$, where the vectors $u_{i}$ are of dimension $m_{3}$, the vectors $v_{i}$ of dimension $m_{1}$ and $w_{i}$ of dimension $m_{1} m_{2}$ are further partitioned into $w_{i}=\left(w_{i}(1), \ldots, w_{i}\left(m_{1}\right)\right)$ of dimension $m_{2}$. The steady-state equations can be written in terms of matrices of lower dimension suitable for numerical implementation as follows:

$$
\begin{gathered}
u_{0}\left[S_{3}+S_{3}^{0} \beta_{3}-\lambda I\right]+v(1) S_{1}^{0} \beta_{3}=0 \\
\lambda u_{i-1}+u_{i}\left[S_{3}-\lambda I\right]=0,1 \leq i \leq N-1, \\
u_{1} S_{3}^{0} \beta_{1}+v_{1} A_{1}+v_{2} S_{1}^{0} \beta_{1}+w_{1}\left[I \otimes S_{2}^{0}\right]=0 \\
u_{i} S_{3}^{0} \beta_{1}+\lambda v_{i-1}+v_{i} A_{1}+v_{i+1} S_{1}^{0} \beta_{1}+w_{i}\left[I \otimes S_{2}^{0}\right]=0,2 \leq i \leq N-1, \\
\lambda u_{N-1} e \beta_{1}+\lambda v_{N-1}+v_{N} A_{1}+v_{N+1} S_{1}^{0} \beta_{1}+w_{N}\left[I \otimes S_{2}^{0}\right]=0, \\
\lambda v_{i-1}+v_{i} A_{1}+v_{i+1} S_{1}^{0} \beta_{1}+w_{i}\left[I \otimes S_{2}^{0}\right]=0, N+1 \leq i \leq n-k \\
\lambda v_{n-k}+v_{n-k+1}\left[A_{1}+\lambda I\right]+w_{n-k+1}\left[I \otimes S_{2}^{0}\right]=0 \\
\mu_{S F} v_{1}\left[I \otimes \beta_{2}\right]+w_{1} A_{2}=0, \\
\mu_{S F} v_{i}\left[I \otimes \beta_{2}\right]+\lambda w_{i-1}+w_{i} A_{2}=0,2 \leq i \leq n-k \\
\mu_{S F} v_{n-k+1}\left[I \otimes \beta_{2}\right]+\lambda w_{n-k}+w_{n-k+1}\left[A_{2}+\lambda I\right]=0 .
\end{gathered}
$$

\subsection{The Steady-State Probability Vectors for the Warm and Hot Systems}

The steady-state equations for the warm and hot systems have more equations than those of the cold system. Suppose that vector $x$ is partitioned as $x=\left(u_{0}, u_{1}, \ldots\right.$, $\left.u_{N-1}, v_{1}, v_{2}, \ldots, v_{n}, w_{1}, w_{2}, \ldots, w_{n}\right)$ to account for the extra states resulting from components failing when the system is down. But first, note that equations (12) through (17), (19) and (20) are common to all three systems. Equations (18) and (21) are replaced with the following set of equations for warm and hot systems found by letting $\tau=\theta$ for the warm system equations and letting $\tau=\lambda$ and $\theta=\lambda$ in $A_{3}$ and $A_{4}$ for the hot system:

$$
\begin{gathered}
\lambda v_{n-k}+v_{n-k+1} A_{3}+v_{n-k+2} S_{1}^{0} \beta_{1}+w_{n-k+2}\left[I \otimes S_{2}^{0}\right]=0 \\
\tau v_{i-1}+v_{i} A_{3}+v_{i+1} S_{1}^{0} \beta_{1}+w_{i}\left[I \otimes S_{2}^{0}\right]=0, n-k+2 \leq i \leq n-1, \\
\tau v_{n-1}+v_{n}\left[A_{3}+\tau I\right]+w_{n}\left[I \otimes S_{2}^{0}\right]=0 \\
\mu_{S F} v_{n-k+1}\left[I \otimes \beta_{2}\right]+\lambda w_{n-k}+w_{n-k+1} A_{4}=0
\end{gathered}
$$




$$
\begin{gathered}
\mu_{S F} v_{i}\left[I \otimes \beta_{2}\right]+\tau w_{i-1}+w_{i} A_{4}=0, n-k+2 \leq i \leq n-1 \\
\mu_{S F^{v}}\left[I \otimes \beta_{2}\right]+\tau w_{n-1}+w_{n}\left[A_{4}+\tau I\right]=0 .
\end{gathered}
$$

Before we state the main results, we need the following lemmas.

\section{Lemma 1:}

$$
\mu_{2} \sum_{i=1}^{n-k+1} w_{i} e=\mu_{S F} \sum_{i=1}^{n-k+1} v_{i} e
$$

where $\mu_{2}^{-1}=-\beta_{2} S_{2}^{-1} e$.

Proof: Adding equations (19), (20) and (21), the stated result follows immediately.

Lemma 2:

$$
\lambda u_{N-1} e+\sum_{i=1}^{N-1} u_{i} S_{3}^{0}=v_{1} S_{1}^{0} .
$$

Proof: This result follows immediately by adding the equations (12) and (13).

\section{Lemma 3:}

$$
\sum_{i=1}^{n-k+1} v_{i} e=\frac{1}{\mu_{1}} \sum_{i=1}^{n-k+1} v_{i} S_{1}^{0}
$$

where $\mu_{1}^{-1}=-\beta_{1} S_{1}^{-1} e$.

Proof: The stated result follows by adding equations (14) through (18), and using Lemma 2 with the fact that

$$
\sum_{i=1}^{n-k+1} w_{i}\left(I \otimes S_{2}^{0}\right)=\mu_{S F} \sum_{i=1}^{n-k+1} v_{i}
$$

Let $X$ denote the time to repair a failed component from the point when the component enters into service. We then have the following result.

Theorem 1: The random variable $X$ is of phase type with representation $(\zeta, U)$ of order $\left(m_{2}+1\right) m_{1}$ where

$$
\zeta=\left(\beta_{1}, 0\right) \text { and } U=\left[\begin{array}{cc}
S_{1}-\mu_{S F} I & \mu_{S F} I \otimes \beta_{2} \\
I \otimes S_{2}^{0} & I \otimes S_{2}
\end{array}\right]
$$

Proof: This result follows immediately by noting that

(a) the initial phase of the repair is chosen according to the probability vector $\alpha$;

(b) an absorption from this set of $m_{1}$ states corresponds to the completion of the repair without any failure on the part of the repairman;

(c) if the repairman fails during the repair time, the Markov chain enters into the set of $m_{1} m_{2}$ states that represent the service time to fix the repairman.

Corollary: The mean time spent in the repair facility by a failed component is given by

$$
\mu_{X}^{\prime}=\mu_{1}^{\prime}\left[1+\mu_{S F} \mu_{2}^{\prime}\right]
$$

where $\mu_{1}^{\prime}$ is the mean repair time of a failed component and $\mu_{2}^{\prime}$ is the mean time to fix the failed server.

Proof: The proof is obtained by using the fact that $\mu_{X}^{\prime}=\left(\pi \boldsymbol{U}^{0}\right)^{-1}$, where $\boldsymbol{U}^{0}$ is 
such that $U \boldsymbol{e}+\boldsymbol{U}^{0}=\mathbf{0}$, and $\pi$ is the solution to $\pi\left(U+\boldsymbol{U}^{0} \boldsymbol{\zeta}\right)=\mathbf{0}$, and $\pi \boldsymbol{e}=1$. By partitioning $\pi=\left(\pi_{1}, \pi_{2}\right)$ and using the special structure of $U$, it can easily be verified that

$$
\pi_{2}=\mu_{S F} \pi_{1}\left(I \otimes \beta_{2}\left(-S_{2}\right)^{-1}\right) \text { and } \pi_{1}=c \beta_{1}\left(-S_{1}\right)^{-1}
$$

where $c$ is the normalizing constant such that $\pi_{1} e+\pi_{2} e=1$. The stated result follows immediately.

Remark: Note that the mean time spent in the repair facility depends only on the failure rate of the server, the mean service time of the unit, and the mean repair time of the server.

Let $Y$ denote the time it takes a failed component to return to working condition for a cold system. Note that $Y$ includes the time, if any, a failed component spends in the queue of the repair facility. We then have the following theorem.

Theorem 2: The random variable $Y$ is of phase type with representation $(\xi, R)$ of order $N m_{3}+(n-k+1)\left[m_{1}+m_{2}\right]$, where the vector $\xi=\left(\boldsymbol{a}_{1}, \ldots, \boldsymbol{a}_{N-1}, \boldsymbol{b}_{1}, \ldots\right.$, $\left.\boldsymbol{b}_{n-k+1}, \mathbf{0}, \boldsymbol{c}_{2}, \ldots, \boldsymbol{c}_{n-k+1}\right)$ is such that $\boldsymbol{a}_{i}=d \boldsymbol{u}_{i-1}, 1 \leq i \leq N-1, \boldsymbol{b}_{1}=d \boldsymbol{u}_{N-1} \boldsymbol{e}$, $\boldsymbol{b}_{i}=d \boldsymbol{v}_{i}, \quad 2 \leq i \leq n-k+1$, and $\boldsymbol{c}_{i}=d \boldsymbol{w}_{i-1}$, for $2 \leq i \leq n-k+1$, with $d=[1-$ $\left.\boldsymbol{v}_{n-k+1} \boldsymbol{e}-\boldsymbol{w}_{n-k+1} \boldsymbol{e}\right]^{-1}$. The matrix $R$ is of the form

$$
R=\left[\begin{array}{ccc}
R_{11} & R_{12} & 0 \\
0 & R_{22} & \mu_{S F}\left(I \otimes \beta_{2}\right) \\
0 & I \otimes S_{2}^{0} & R_{33}
\end{array}\right],
$$

where the entries of $R$ are

$$
\begin{aligned}
& R_{11}=\left[\begin{array}{cccccc}
S_{3}-\lambda I & \lambda I & 0 & \ldots & 0 & 0 \\
0 & S_{3}-\lambda I & \lambda I & \ldots & 0 & 0 \\
\vdots & \vdots & \vdots & \ldots & \vdots & \vdots \\
0 & 0 & 0 & \ldots & S_{3}-\lambda I & \lambda I \\
0 & 0 & 0 & \ldots & 0 & S_{3}-\lambda I
\end{array}\right], \\
& R_{12}=\left[\begin{array}{ccccc}
0 & 0 & 0 & \ldots & 0 \\
0 & 0 & 0 & \ldots & 0 \\
\vdots & \vdots & \vdots & \ldots & \vdots \\
0 & 0 & 0 & \ldots & 0 \\
\lambda I & 0 & 0 & \ldots & 0
\end{array}\right] \\
& R_{22}=\left[\begin{array}{cccccc}
A_{1} & \lambda I & 0 & \ldots & 0 & 0 \\
S_{1}^{0} \beta_{1} & A_{1} & \lambda I & \ldots & 0 & 0 \\
0 & S_{1}^{0} \beta_{1} & A_{1} & \ldots & 0 & 0 \\
\vdots & \vdots & \vdots & \ldots & \vdots & \vdots \\
0 & 0 & 0 & \ldots & S_{1}^{0} \beta_{1} & A_{1}+\lambda I
\end{array}\right]
\end{aligned}
$$




$$
R_{33}=\left[\begin{array}{ccccc}
A_{2} & \lambda I & 0 & \ldots & 0 \\
0 & A_{2} & \lambda I & \ldots & 0 \\
\vdots & \vdots & \vdots & \ldots & \vdots \\
0 & 0 & 0 & \ldots & \lambda I \\
0 & 0 & 0 & \ldots & A_{2}+\lambda I
\end{array}\right]
$$

Proof: The theorem follows by noting that a failed component will see the server either

(a) on vacation with $i(1 \leq i \leq N)$ components (including the current one) waiting for repair (note that when $i=N$ the server will be recalled from vacation instantaneously), or

(b) busy with $i(1 \leq i \leq n-k+1)$ components (including the current one) in repair facility,

(c) is under repair with $i(1 \leq i \leq n-k+1)$ components (including the current one) in the repair facility.

Since failure times of the components are exponential, the probability vector $\boldsymbol{\xi}$ is just a scalar multiple of the relevant probabilities at an arbitrary time.

Let $Z$ denote the time it takes the system to fail starting with all functioning components. First note that the probability density function of $Z$ is identical for all three systems (cold, warm, hot). The following theorem shows that $Z$ is of phase type.

Theorem 3: The random variable $Z$ is of phase type with representation $(\boldsymbol{\eta}, L)$ of order $\mathrm{Nm}_{3}+(n-k)\left[m_{1}+m_{2}\right]$, where $\boldsymbol{\eta}=\left(\boldsymbol{\beta}_{3}, \mathbf{0}\right)$ and the matrix $L$ is of the form

$$
L=\left[\begin{array}{ccc}
L_{11} & L_{12} & 0 \\
L_{21} & L_{22} & \mu_{S F}\left(I \otimes \beta_{2}\right) \\
0 & I \otimes S_{2}^{0} & L_{33}
\end{array}\right],
$$

where $L_{11}=B_{11}^{c}$ and $L_{12}$ is of the form $B_{12}^{c}$ with dimension $N m_{3} \times(n-k) m_{1}$ and $L_{21}$ is of the form $B_{21}^{c}$ with dimension $(n-k) m_{1} \times N m_{3}$, and

$$
\begin{aligned}
& L_{22}=\left[\begin{array}{cccccc}
A_{1} & \lambda I & 0 & \ldots & 0 & 0 \\
S_{1}^{0} \beta_{1} & A_{1} & \lambda I & \ldots & 0 & 0 \\
0 & S_{1}^{0} \beta_{1} & A_{1} & \ldots & 0 & 0 \\
\vdots & \vdots & \vdots & \ldots & \vdots & \vdots \\
0 & 0 & 0 & \ldots & S_{1}^{0} \beta_{1} & A_{1}
\end{array}\right] \\
& L_{33}=\left[\begin{array}{ccccc}
A_{2} & \lambda I & 0 & \ldots & 0 \\
0 & A_{2} & \lambda I & \ldots & 0 \\
\vdots & \vdots & \vdots & \ldots & \vdots \\
0 & 0 & 0 & \ldots & \lambda I \\
0 & 0 & 0 & \ldots & A_{2}
\end{array}\right]
\end{aligned}
$$


Proof: The results follow immediately by noting that the set of states $(\boldsymbol{n}-\boldsymbol{k}+1)$ and $(\boldsymbol{n}-\boldsymbol{k}+\mathbf{1})^{\prime}$ correspond to the system failing, and then, combining these into one absorbing state.

Suppose $V_{1}$ denotes the vacation duration of the server. Then we have

Theorem 4: The random variable $V_{1}$ is of phase type with representation $\left(\varphi, B_{11}^{c}\right)$ of order $\mathrm{Nm}_{3}$, where the vector $\varphi=\left(\boldsymbol{\beta}_{3}, \mathbf{0}\right)$.

Proof: This follows by noting the vacation period of the server starts when all components are in functioning state and returns according to $(N, T)$-policy.

Remark: Note that, as is expected, the random variable $V_{1}$ depends only on the components' failure rate $\lambda$ and the server's vacation time $T$.

Corollary: The mean vacation period of the server is given by

$$
\mu_{V}^{\prime}=\lambda \frac{\sum_{i=0}^{N-1} u_{i} e}{u_{0} e}
$$

where the steady-state probabilities, $u_{i}, 0 \leq i \leq N-1$, are as given in (12)-(14).

Proof: The result is obtained by using the fact that $\mu_{V}^{\prime}=\left(\pi B^{0}\right)^{-1}$, where $B^{0}$ is such that $B_{11}^{c} e+B^{0}=0$, and $\pi$ is the solution to $\pi\left(B_{12}^{c}+B^{0} \varphi\right)=0$, and $\pi e=1$. Partitioning $\pi=\left(\pi_{0}, \pi_{1}, \ldots, \pi_{N-1}\right)$, and noting that $\pi_{i}=d u_{i}, 0 \leq i \leq N-1$ with $d$ is such that $\pi e=1$, the stated result follows immediately.

Suppose that $V_{2}$ denotes the non-vacation duration of the server. Note that this duration includes the time the server is busy fixing failed components as well as the time the server is being repaired. Then we have:

Theorem 5: The random variable $V_{2}$ is of phase type with representation $\left(\psi \otimes \beta_{1}, M\right)$ of order $(n-k+1)\left[m_{1}+m_{2}\right]$, where the vector $\psi=\left(\psi_{1}, \ldots, \psi_{N}, 0, \ldots\right.$, $0)$ is given by

$$
\psi_{i}=d u_{i} S_{3}^{0}, 1 \leq i \leq N-1, \text { and } \psi_{N}=d u_{N-1} e
$$

where $d$ is the normalizing constant and the matrix $M$ is

$$
M=\left[\begin{array}{cc}
B_{22}^{c} & \mu_{S F}\left(I \otimes \beta_{2}\right) \\
I \otimes S_{2}^{0} & B_{33}^{c}
\end{array}\right],
$$

where $B_{22}^{c}$ and $B_{33}^{c}$ are as given in (4) and (5).

Proof: This result follows by noting that the non-vacation period of the server starts with at least one failed component waiting for repair and with the return of the server under $(N, T)$-policy.

The following interesting result gives an expression for the fraction of time the server is under repair.

Theorem 6: The fraction of time the server is under repair is given by

$$
P(\text { server is under repair })=\frac{p_{N V} \mu_{S F}}{\mu_{S F}+\mu_{2}},
$$

where $\mu_{2}$ is the repair rate of the failed server, and $p_{N V}$ is the probability that the server is not on vacation where 


$$
p_{N V}=1-\sum_{i=0}^{N-1} u_{i} e .
$$

Proof: This follows immediately from Lemma 1.

Remark: The result (28) can be intuitively explained as follows. First note that the failure of the server may occur only during a non-vacation period. Since a failed server is immediately started on a phase type service, the fraction of time the server is under repair is equal to the product of the probability that the server is not on vacation and the probability of the server being busy in an $\mathrm{M} / \mathrm{PH} / 1$ queue with no waiting room. The arrival rate is given by $\mu_{S F}$ and the service time is of phase type with representation $\left(\boldsymbol{\beta}_{2}, S_{2}\right)$.

Theorem 7: The probability mass function $\left\{a_{i}\right\}$ of the number of failed components waiting for repair at the time when the vacationing server returns to the system, and the conditional probability mass function $\left\{b_{i}\right\}$ of the number of failed components waiting for repair given that the server returning from vacation finds at least one component in the repair facility are given by

$$
\begin{gathered}
a_{i}=g G^{i} S_{3}^{0}, 0 \leq i \leq N-1, a_{N}=\lambda g G^{N-1} e \\
b_{i}=d a_{i}, 1 \leq i \leq N
\end{gathered}
$$

where

$$
g=\beta_{3}\left(\lambda I-S_{3}\right)^{-1}, \quad G=\lambda\left(\lambda I-S_{3}\right)^{-1}, \text { and } d=\left(\beta_{3} G e\right)^{-1} \text {. }
$$

Proof: First note that $u_{i} S_{3}^{0} d t, 1 \leq i \leq N$ gives the steady-state probability that in a small time interval of width $\overline{d t}$, the server finishes the vacation to find $i$ components waiting in the repair facility. The stated result follows from equations (12) through (14) and Lemma 2.

Remark: Note that the probabilities in (29), as is to be expected, depend only on the components' failure rate and the distribution of the server's vacation period.

\section{System Performance Measures}

In this section we give a number of system performance measures useful in qualitative interpretation of the model under study. These measures are for a cold system and similar measures are obtained for the other two systems. The details are omitted. However, in the numerical example section we will compare these measures for all three systems.

(a) The fraction of time the server is on vacation is given by $\sum_{i=0}^{N-1} u_{i} e$.

(b) The fraction of the time the server is under repair is $\sum_{i=1}^{n+k} w_{i} e$. Note that this measure is given in terms of $\mu_{S F}$ and $\mu_{2}$ from equation (28).

(c) The probability mass function of the number of components under repair is given by

$$
f_{R}(i)=\left\{\begin{array}{cc}
u_{0} e, & i=0 \\
u_{i} e+v_{i} e+w_{i} e, & 1 \leq i \leq N-1 \\
v_{i} e+w_{i} e, & N \leq i \leq n-k+1 .
\end{array}\right.
$$


The mean and the standard deviation of this probability function can also be computed.

(d) The fraction of time the system is down is given by $u_{n-k+1} e+w_{n-k+1} e$.

(e) The mean time spent in the repair facility by a failed component is given by $(25)$.

(f) The mean vacation duration of the server is given by (27).

(g) The mean non-vacation period of the server is calculated as $\left(\psi \otimes \beta_{1}\right)$ $(-M)^{-1} e$.

(h) The mean number of components waiting for repair when the server returns from a vacation and the conditional mean number of components waiting for repair given that the server returning from vacation sees at least one component in the repair facility can be computed from the probability functions given in (29).

\section{Numerical Examples}

In this section we discuss some interesting numerical examples and present an optimization problem. A Fortran code was developed and tested on a large number of examples using a Pentium II PC. Particular cases, which reduce to simple models and internal accuracy checks, such as the one outlined in Section 2 and Lemma 1, are used to confirm the correctness of the code. Recall that $\mu_{i}^{\prime}=\beta_{i}\left(-S_{i}\right)^{-1} e, 1 \leq i \leq 3$, is the mean of the PH-distribution with representation $\left(\beta_{i}, S_{i}\right)$.

For illustrative purpose, we consider the following three PH-distributions for repair times of the components, for vacation duration of the server, and for the fixing times of the failed server:

Exponential (10) (EX),

Erlang with 10 phases and with parameter 10 (ER), and

Hyperexponential: $0.90 \operatorname{EXP}(100)+0.09 \operatorname{EXP}(1)+0.01 \operatorname{EXP}(0.1)(\mathbf{H E})$.

The above distributions are normalized so that $\mu_{1}^{\prime}=0.5, \mu_{2}^{\prime}=0.125$, and $\mu_{3}^{\prime}=0.25$. The other parameters are taken to be $n=10, k=4, \lambda=1, \theta=0.3$, and $\mu_{S F}=1$. We consider 27 possible combinations for the reliability model under study by choosing one of these three distributions for the repair times of the components, one for the fixing times of the failed server and one for the server's vacation period. The five performance measures

(a) the mean time to system failure,

(b) the mean vacation period of the server,

(c) the mean non-vacation period of the server,

(d) the probability the server is on vacation and

(e) the mean number of components under repair

are plotted in Figures 1 through 16 as functions of $N$ varying from 1 through $n-k=6$. The two performance measures

$(f)$ the probability mass function of the number of failed components seen by the server returning from vacation, and

(g) the conditional probability mass function of the number of failed components seen by the returning server given that at least one failed component in the repair facility,

are plotted in Figures 17 through 18. On examination of these figures, we notice the following observations. 
The Mean Time to System Failure (Figures 1 through 3):

- As expected the mean time to system failure decreases as $N$ increases for all cases.

- By fixing the two distributions as ER and varying the other with one of the three distributions (ER, EX, or $\mathbf{H E}$ ), we notice that this measure tends to decrease with increasing variability. In Figure 1, we plot this performance measure by varying the server's vacation period.

The Mean Vacation Period of the Server (Figure 4):

- Recall that the mean vacation period of the server depends only on $\lambda$ and the distribution of the vacation period. As expected, this measure is identical for all three distributions when $N=1$. However, for other values of $N$ this measure increases with increasing variance of the vacation period and also with $N$.

- $\quad$ For hyperexponential case, this measure varies significantly as $N$ is varied. However, for the other two cases, when $N \geq 2$, the change in this measure appears to be insignificant.

The Mean Non-Vacation Period of the Server (Figures 5-10):

- By varying the repair times of the components with one of the three distributions (ER, EX, or HE) and fixing the other two with ER, we notice that the mean non-vacation period of the server appears to decrease with increasing variability (see Figure 5). Furthermore, the hyperexponential distribution appears to yield smaller means for all values of $N$ compared to the other two cases.

- By varying the fixing times of the failed server with one of the three distributions (ER, EX, or HE), and fixing the other two with ER, w notice that this measure decreases with increasing variability (see Figure 6). Going from $N=1$ to $N=2$, there is a significant jump in the mean for all three distributions. For other values of $N$, there appears to be insignificant change.

- By varying the vacation period with one of the three distributions (ER, EX, or HE) and fixing the other two as ER, we notice that this measure increases with increasing variability (see Figure 7). This observation is in direct contrast with the previous two observations.

- Suppose we use HE instead of ER to fix the other two distributions and vary the other with one of ER, EX, or HE. While the behavior (see Figures 8 and 10) of this measure is very similar to the earlier ones in the cases when the repair times of the components and when the vacation period the server is varied, an interesting observation (see Figure 9) is seen in the case when the fixing times of the failed server is varied. Here, we note that this measure appears to be independent of the distribution.

The Probability that the Server is on Vacation (Figures 11-13):

- By varying the repair times of the components with one of the three distributions (ER, EX, or HE), and fixing the other two as ER, we notice that the probability that the server is on vacation appears to increase with increasing variability (see Figure 11). Furthermore, this measure appears to be independent of the values of $N$. The same observation holds when the fixing time of the failed server is varied and the other two fixed to be ER (see Figure 12). 
- In the case when the vacation period of the server is varied with one of the the three distributions (ER, EX, or HE) and fixing the other two as ER, we notice that this measure increases with increasing variance. In the HE case, we notice that the probability increases as $N$ increases.

\section{The Mean Number of Components Under Repair (Figures 14-16):}

- here, we look at the three systems: cold, warm, and hot by fixing two of the three distributions as ER and the other with HE. When the vacation period is $\mathbf{H E}$, the mean number of components under repair appears to be almost the same for all three systems (see Figure 16); however, we see a significant difference in this measure for the other two cases (see Figures 14 and 15). As expected, this measure is largest for hot system and smallest for the cold system.

The Probability Functions of the Number of Failed Components (Figures 17-18):

- In Figure 17, the probability mass function of the number of failed components seen by the server returning from vacation, is plotted for the three distributions for the case when $N=6$. We notice that the probability that the server sees no component waiting for repair is highest for the $\mathbf{H E}$ case. This can be intuitively explained as follows. When the vacation period is $\mathbf{H E}$, the server will have a large number of small vacation periods followed by a long vacation period. During the shorter vacation periods, the server is more likely to see no components waiting for repair.

- In Figure 18, the conditional probability mass function of the number of failed components seen by the returning server, conditional on the fact that at least one failed component is waiting for repair, is plotted for the case when $N=6$. The figure indicates, as expected, that an $\mathbf{H E}$ vacation is more likely to see larger components waiting for repair than the other two distributions.

\section{References}

[1] Akhtar, S., Reliability of $k$-out-of- $n$ : $G$ systems with imperfect fault coverage, IEEE Trans. Reliability 43 (1994), 101-106.

[2] Balaban, H.S., Some effects of redundancy on system reliability, Proc. 6th National Symp. Reliability and Quality Control (1960), 89-95.

[3] Barlow, R.E. and Proschan, F., Mathematical Theory of Reliability, John Wiley and Sons, New York 1965.

[4] Barlow, R.E. and Proschan, F., Statistical Theory of Reliability and Life Testing, Holt, Rihehart, and Winston, Inc. 1975.

[5] Bellman, R.E., Introduction to Matrix Analysis, McGraw Hill, New York 1960.

[6] Chakravarthy, S.R. and Agarwal, A., Analysis of a machine repair problem with an unreliable server and phase type repairs and services, submitted.

[7] Kapur, K.C. and Lamberson, L.R., Reliability in Engineering Design, John Wiley and Sons 1977.

[8] Ke, J.C. and Wang, K.H., Cost analysis of the M/M/R machine repair problem with balking, reneging, and server breakdowns, J. of the Oper. Res. Soc. 50 (1999), 275-282.

[9] Kenyon, R.L. and Newell, R.L., Steady-state availability of $k$-out-of- $n$ : $G$ system with single repair, IEEE Trans. Reliability R-32 (1983), 188-190. 
[10] Kullstam, P.A., Availability, MTBF, MTTR for repairable $m$-out-of- $n$ system, IEEE Trans. Reliability R-30 (1981), 393-394.

[11] Liu, B. and Cao, J., Machine service model with a service station consisting of $r$ unreliable units, Microelectronics and Reliability 35 (1995), 683-690.

[12] Liu, H., Reliability of a load-sharing of $k$-out-of- $n$ : $G$ system: non-idle components with arbitrary distributions, IEEE Trans. Reliability 47 (1993), 279-284.

[13] Nakagawa, T., Optimization problems in $k$-out-of- $n$ systems, IEEE Trans. Reliability R-34 (1985), 248-250.

[14] Neuts, M.F., Probability distributions of phase type, In: Liber Amicorum Prof. Emeritus H. Florin, Dept. of Math., Univ. of Louvain (1975), 173-206.

[15] Pham, H., On the optimal design of $k$-out-of- $n: G$ subsystems with single repair, IEEE Trans. Reliability 41 (1992), 572-574.

[16] Pham, H., Optimal design of $k$-out-of- $n$ redundant systems, Microelectronics and Reliability 32 (1992), 119-126.

[17] Philips, M.J., $k$-out-of- $n: G$ systems are preferable, IEEE Trans. Reliability R-29 (1980), 166-169.

[18] Robinson, D.G. and Neuts, M.F., An algorithmic approach to increased reliability through standby redundancy, IEEE Trans. Reliability 38 (1989), 430435.

[19] Shao, J. and Lamberson, L.R., Modeling a shared-load $k$-out-of- $n: G$ system, IEEE Trans. Reliability 40 (1991), 205-209.

[20] Shooman, M.L., Probabilistic Reliability: An Engineering Approach, McGrawHill 1968.

[21] Srinivasa Rao, T.S.S. and Gupta, U.C., Performance modeling of the M/G/1 machine repairman problem with cold-, warm- and hot-standbys, Computers and Ind. Eng. 38 (2000), 251-257.

[22] Wang, K., Profit analysis of the machine-repair problem with a single service station subject to breakdowns, J. of the Oper. Res. Soc. 41 (1990), 1153-1160.

[23] Wang, K., Profit analysis of the $M / M / R$ machine repair problem with spares and server breakdowns, J. of the Oper. Res. Soc. 45 (1994), 539-548.

[24] Wang, K., Profit analysis of the machine repair problem with cold standbys and two modes of failure, Microelectronics and Reliability 34 (1994), 1635-1642.

[25] Wang, K. and Hsu, L.Y., Cost analysis of the machine-repair problem with $R$ non-reliable service stations, Microelectronics and Reliability 35 (1995), 923-934.

[26] Wang, K. and Kuo, M., Profit analysis of the $M / E_{k} / 1$ machine repair problem with a non-reliable service station, Computers and Ind. Eng. 32 (1997), 587-594.

[27] Wang, K. and Lee, H., Cost analysis of the cold-standby M/M/R machine repair problem with multiple modes of failure, Microelectronics and Reliability 38 (1998), 435-441.

[28] Wang, K. and Sivazlian, B.D., Cost analysis of the $M / M / R$ machine repair problem with spares operating under variable service rates, Microelectronics and Reliability 32 (1992), 1171-1183.

[29] Wartenhorst, P.N., Parallel queueing systems with server breakdowns and repair, European J. of Oper. Res. 82 (1995), 302-322. 


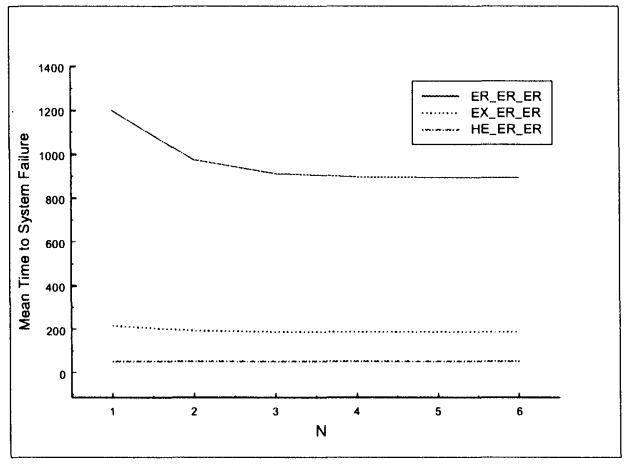

Figure 1

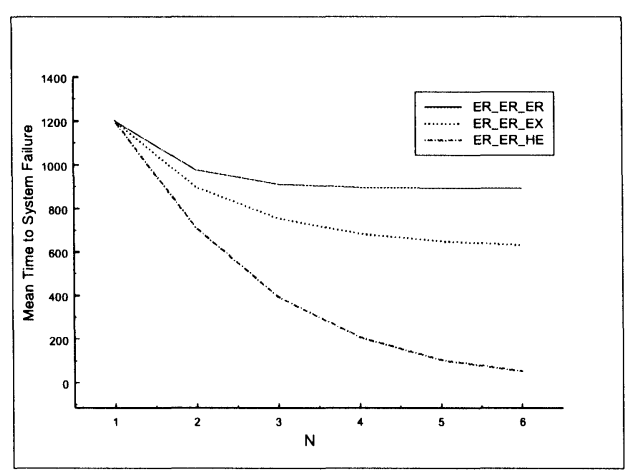

Figure 3

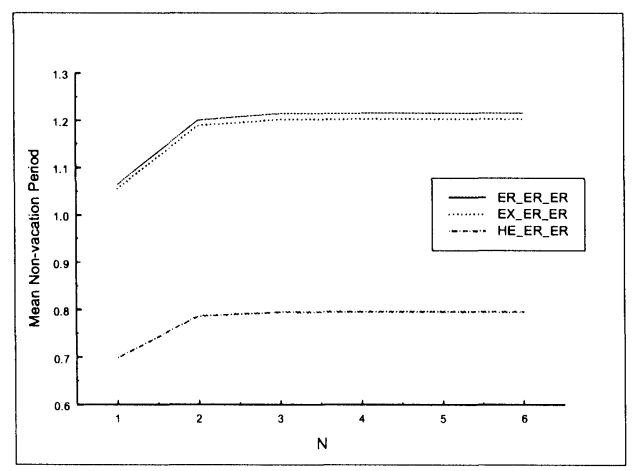

Figure 5

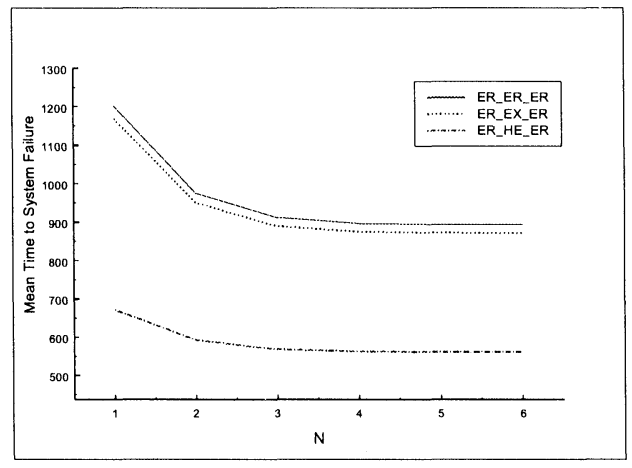

Figure 2

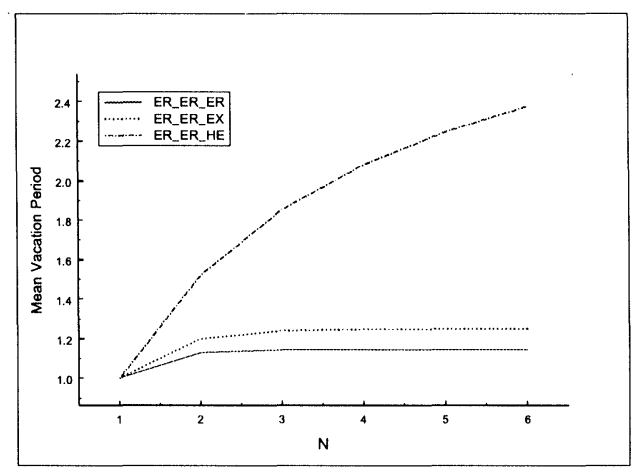

Figure 4

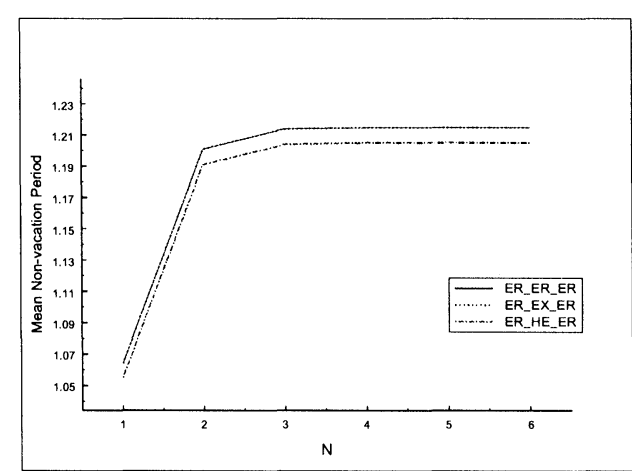

Figure 6 


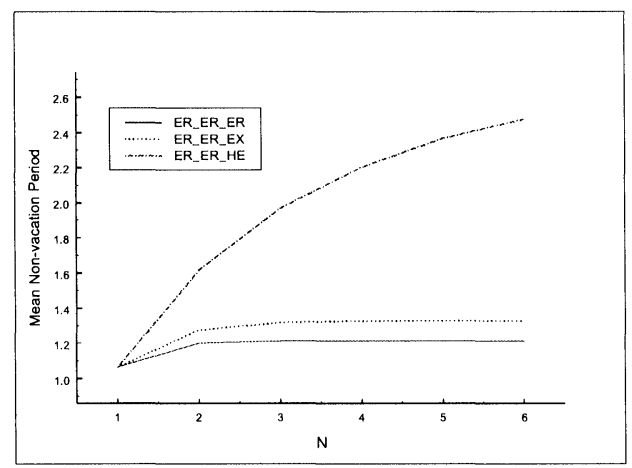

Figure 7

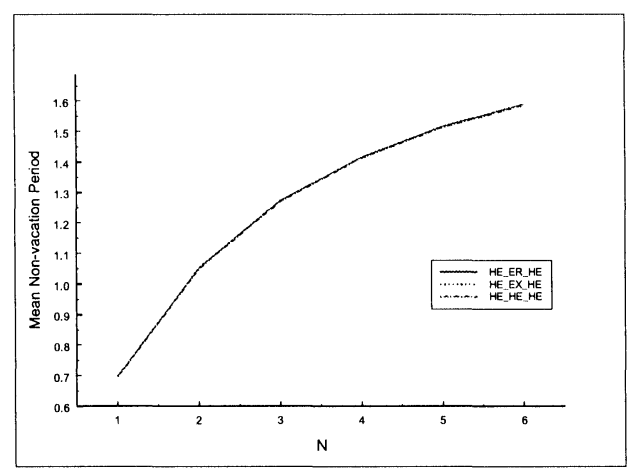

Figure 9

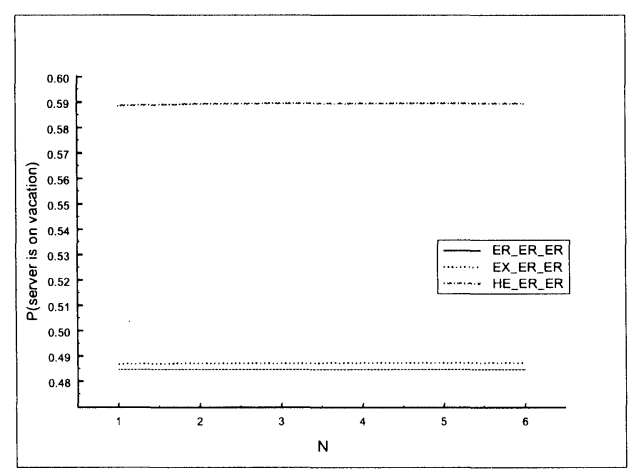

Figure 11

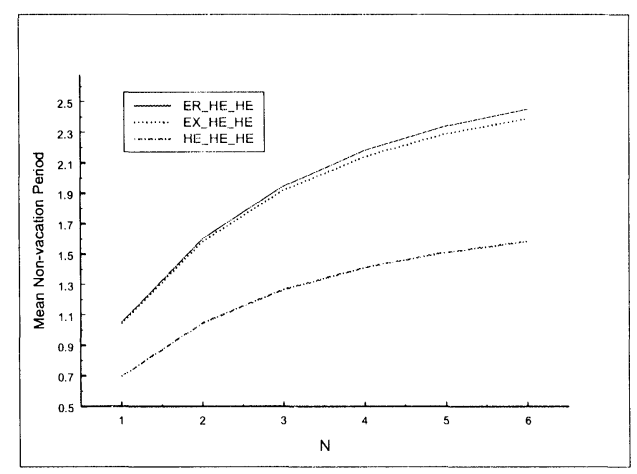

Figure 8

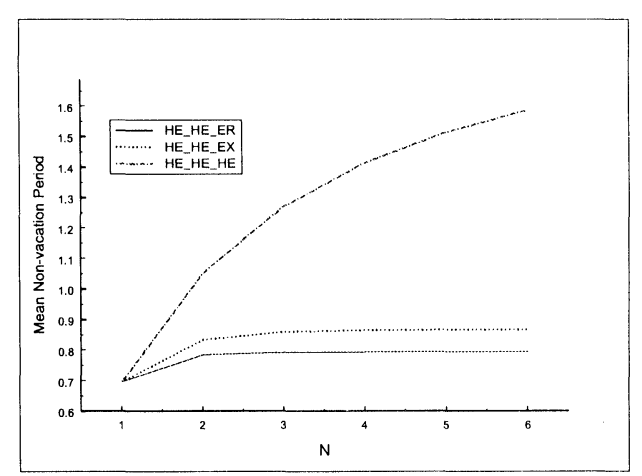

Figure 10

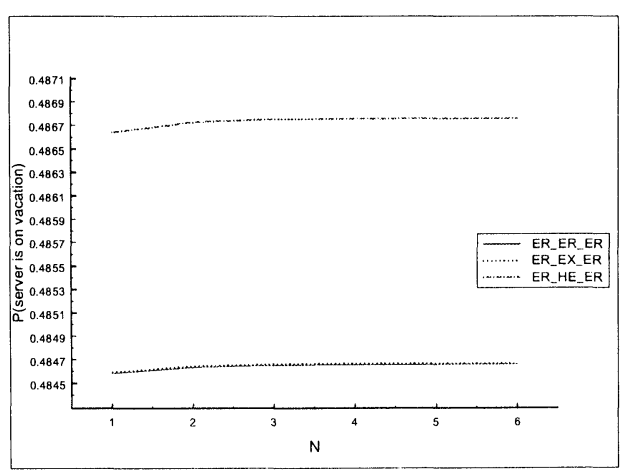

Figure 12 


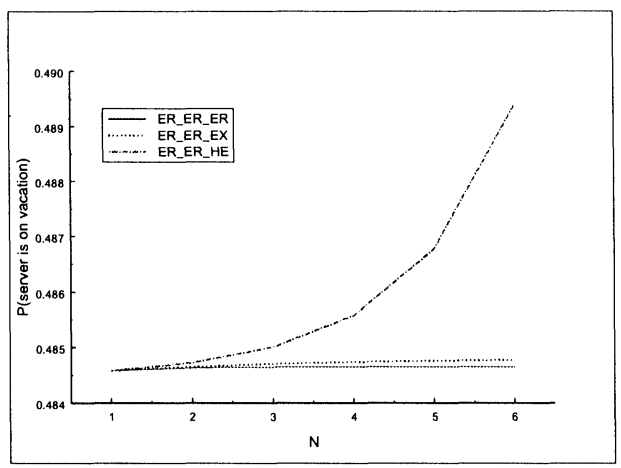

Figure 13

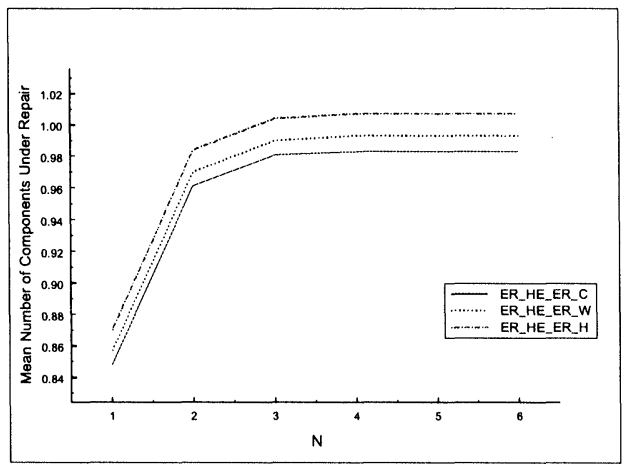

Figure 15

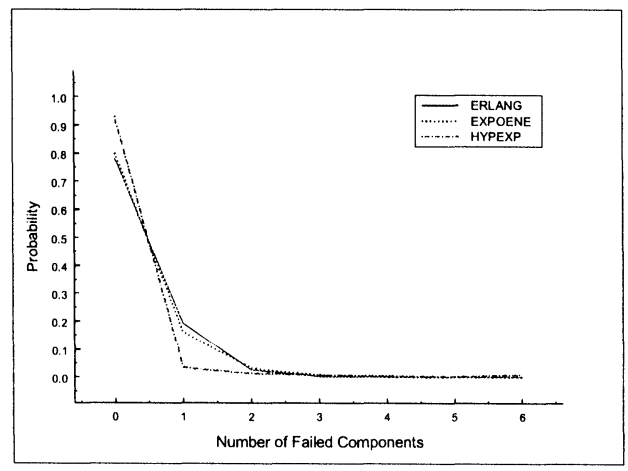

Figure 17

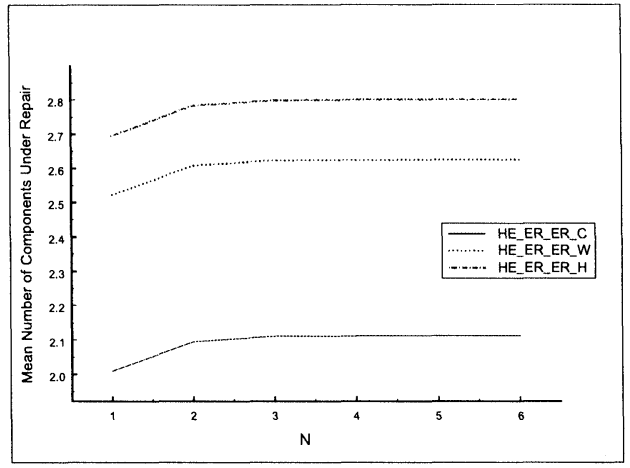

Figure 14

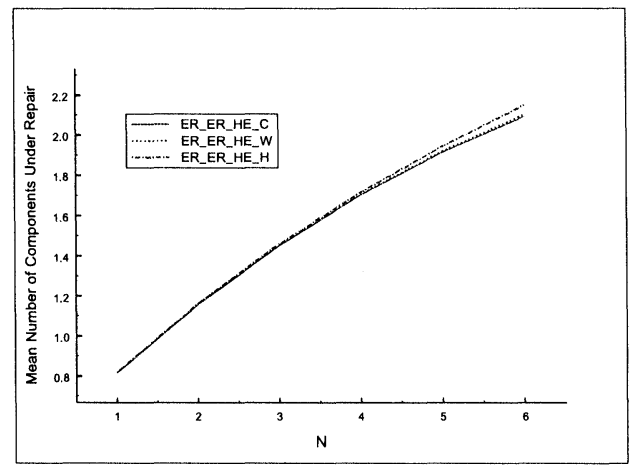

Figure 16

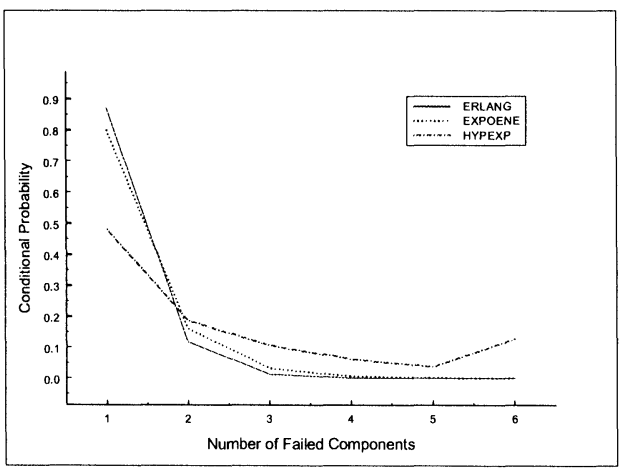

Figure 18 


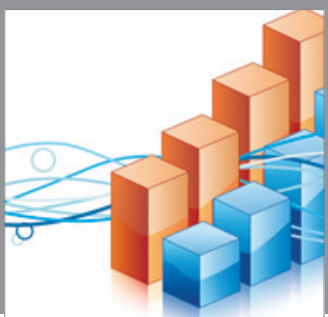

Advances in

Operations Research

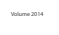

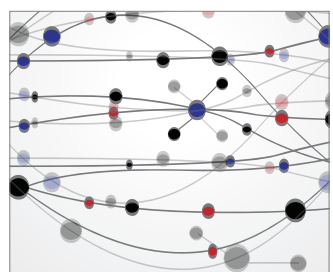

\section{The Scientific} World Journal
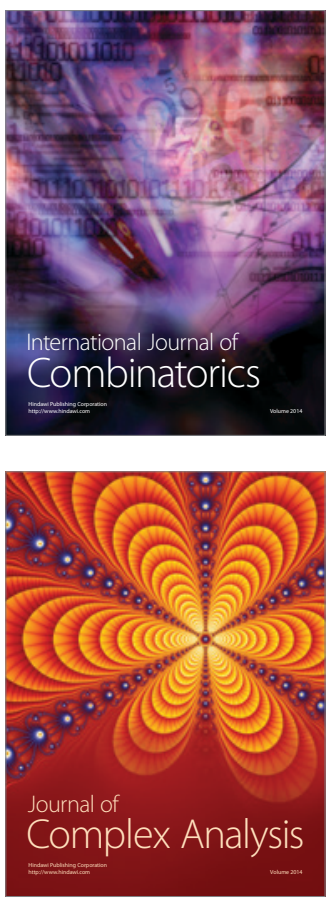

International Journal of

Mathematics and

Mathematical

Sciences
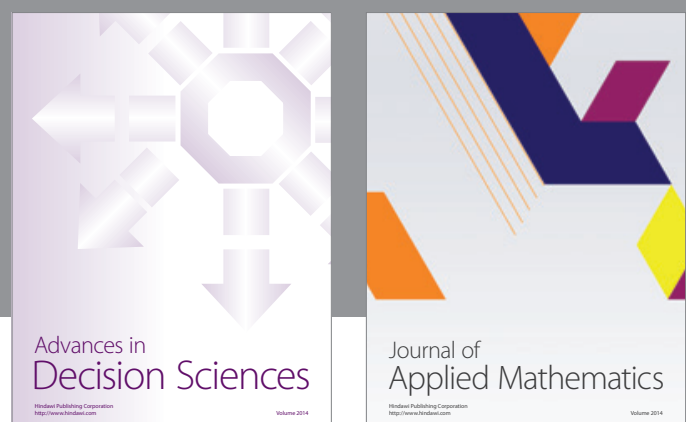

Journal of

Applied Mathematics
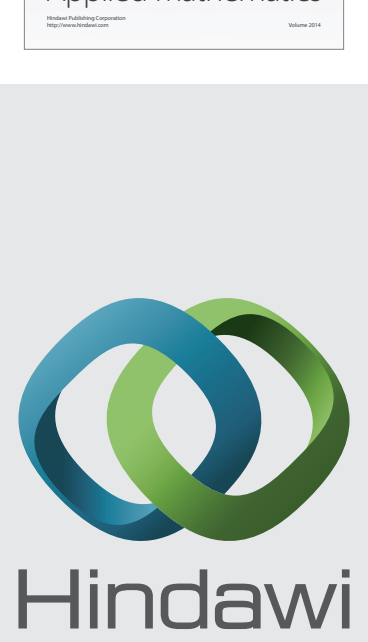

Submit your manuscripts at http://www.hindawi.com
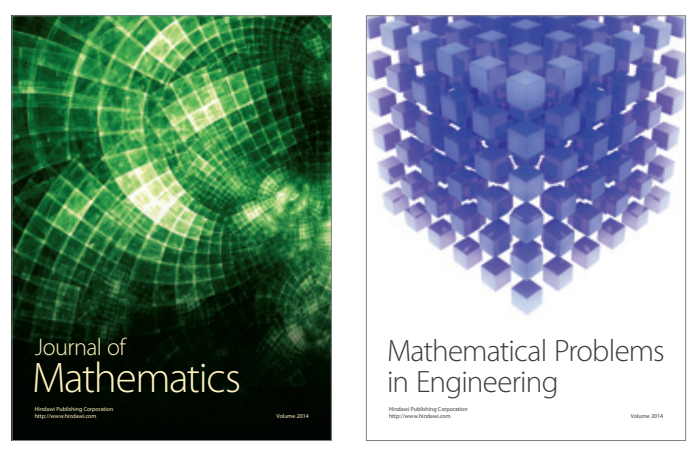

Mathematical Problems in Engineering
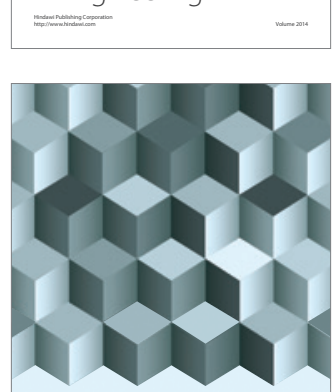

Journal of

Function Spaces
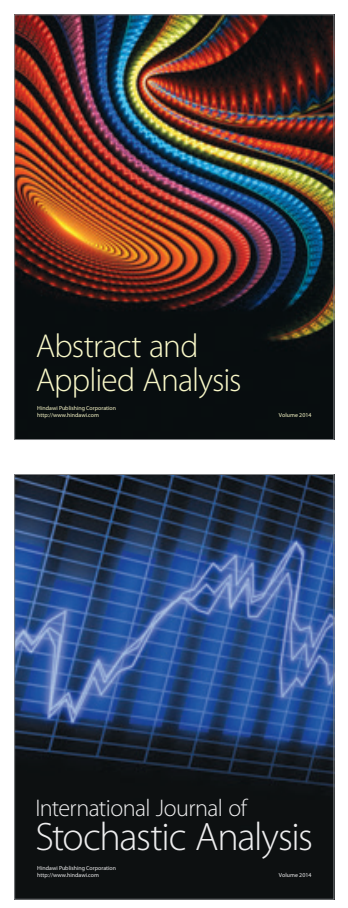

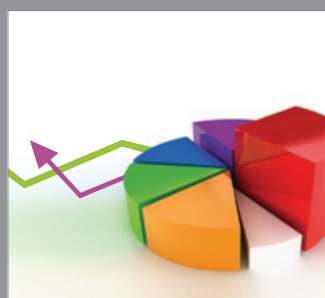

ournal of

Probability and Statistics

Promensencen
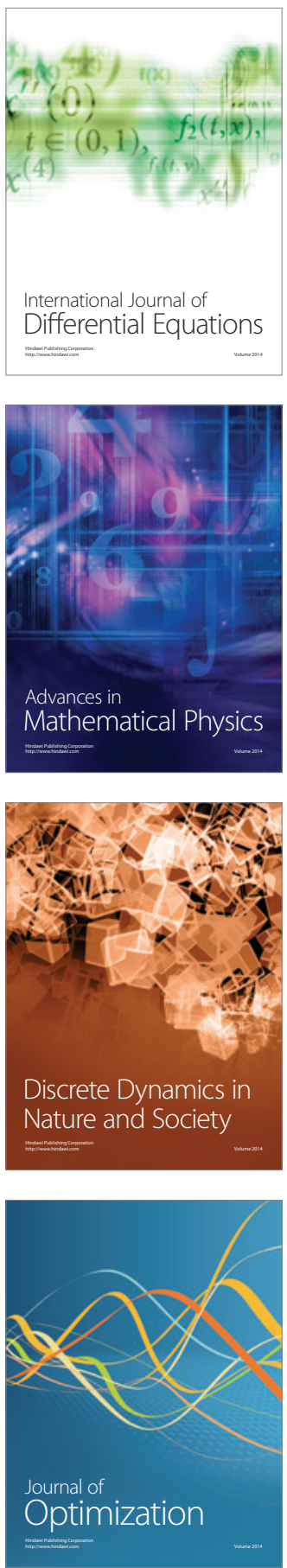